\title{
Multiparametric imaging hippocampal neurodegeneration and functional connectivity with simultaneous PET/MRI in Alzheimer's disease
}

\author{
Shaozhen Yan ${ }^{1,2,3} \cdot$ Chaojie Zheng ${ }^{2} \cdot$ Bixiao Cui ${ }^{4} \cdot$ Zhigang Qi $^{1,3} \cdot$ Zhilian Zhao $^{1,3} \cdot$ Yanhong An $^{1,3} \cdot$ Liyan $_{\text {Qiao }}{ }^{5}$ \\ Ying $\mathrm{Han}^{6} \cdot$ Yun Zhou ${ }^{2} \cdot$ Jie Lu $\mathrm{Lu}^{1,3,4}$ (i)
}

Received: 20 December 2019 / Accepted: 3 March 2020 / Published online: 10 March 2020

(C) The Author(s) 2020

\begin{abstract}
Purpose The objective of this study is to investigate the hippocampal neurodegeneration and its associated aberrant functions in mild cognitive impairment (MCI) and Alzheimer's disease (AD) patients using simultaneous PET/MRI.

Methods Forty-two cognitively normal controls (NC), $38 \mathrm{MCI}$, and 22 AD patients were enrolled in this study. All subjects underwent ${ }^{18}$ F-FDG PET/functional MRI (fMRI) and high-resolution T1-weighted MRI scans on a hybrid GE Signa PET/ MRI scanner. Neurodegeneration in hippocampus and its subregions was quantified by regional gray matter volume and

${ }^{18}$ F-FDG standardized uptake value ratio (SUVR) relative to cerebellum. An iterative reblurred Van Cittert iteration method was used for voxelwise partial volume correction on ${ }^{18}$ F-FDG PET images. Regional gray matter volume was estimated from voxel-based morphometric analysis with MRI. fMRI data were analyzed after slice time correction and head motion correction using statistical parametric mapping (SPM12) with DPARSF toolbox. The regions of interest including hippocampus, cornu ammonis (CA1), CA2/3/dentate gyrus (DG), and subiculum were defined in the standard MNI space.

Results Patient groups had reduced SUVR, gray matter volume, and functional connectivity compared to NC in CA1, CA2/3/ $\mathrm{DG}$, and subiculum $(\mathrm{AD}<\mathrm{MCI}<\mathrm{NC})$. There was a linear correlation between the left $\mathrm{CA} 2 / 3 \mathrm{DG}$ gray matter volume and ${ }^{18} \mathrm{~F}$ FDG SUVR in AD patients $(P<0.001, r=0.737)$. Significant correlation was also found between left CA2/3/DG-superior medial frontal gyrus functional connectivity and left CA2/3/DG hypometabolism in patients with AD. The functional connectivity of right CA1-precuneus in patients with $\mathrm{MCI}$ and right subiculum-superior frontal gyrus in patients with AD was positively correlated with mini mental status examination scores $(P<0.05)$.

Conclusion Our findings demonstrate that the associations existed at subregional hippocampal level between the functional connectivity measured by fMRI and neurodegeneration measured by structural MRI and ${ }^{18}$ F-FDG PET. Our results may provide a basis for precision neuroimaging of hippocampus in AD.
\end{abstract}

Keywords Alzheimer's disease · Hippocampal subregions · Neurodegeneration · Hybrid PET/MRI · Voxel-based morphometric analysis

This article is part of the Topical Collection on Neurology

Yun Zhou

yunzhou@wustl.edu

$\triangle$ Jie Lu

imaginglu@hotmail.com

1 Department of Radiology, Xuanwu Hospital, Capital Medical University, Beijing, China

2 Mallinckrodt Institute of Radiology, Washington University School of Medicine, 510 Kingshighway Blvd., St. Louis, MO, USA
3 Beijing Key Laboratory of Magnetic Resonance Imaging and Brain Informatics, Beijing, China

4 Department of Nuclear Medicine, Xuanwu Hospital, Capital Medical University, Beijing, China

5 Department of Neurology, Yuquan Hospital, Clinical Neuroscience Institute, Medical Center, Tsinghua University, Beijing, China

6 Department of Neurology, Xuanwu Hospital, Capital Medical University, Beijing, China 


\section{Introduction}

The hippocampus, as a structure playing a key role in cognitive processes, is known to remain central to the understanding of the Alzheimer's disease (AD) pathophysiology with sensitivity to the neurofibrillary tangle development and a strong association with progression to $\mathrm{AD}$ [1-3]. It is widely recognized that the hippocampus is heterogeneous and can be divided into subregions with different functions and vulnerabilities to neurodegenerative diseases [4-6]. While the hippocampal subregions are thought to exhibit distinct functions, the neural substrate for aberrant functionalities remains elusive.

Several imaging studies have identified and investigated hippocampal subregions with functional MRI (fMRI) in healthy young and aging human brain $[7,8]$. Using in vivo MRI, the hippocampus can be divided into three subregions: cornu ammonis (CA1), CA2/3/dentate gyrus (CA2/3/DG), and subiculum [9]. Resting-state fMRI studies showed that the disrupted total hippocampal connectivity [10], right CA1 and left CA2 subregions connectivity [11], and subiculum network (functional connectivity with frontal and posterior cingulate cortex [PCC] regions) [12] in mild cognitive impairment $(\mathrm{MCI})$ and $\mathrm{AD}$ patients were strongly associated with memory impairment $[5,13,14]$. In addition to fMRI, the fluorine $18\left({ }^{18} \mathrm{~F}\right)$ fluorodeoxyglucose $\left({ }^{18} \mathrm{~F}\right.$-FDG) PET showed that the glucose metabolism of the left hippocampal body CA2/3 and CA4/DG subregions was significantly lower in the early $\mathrm{AD}$ group than in the control group [15]. In MCI patients, we found that the left hippocampal CA2 functional connectivity measured by resting-state fMRI was associated with decreased dorsal raphe nuclei binding potential measured by $\left[{ }^{11}\right.$ C]DASB PET [16].

The previous structural MRI, fMRI, and ${ }^{18}$ F-FDG PET studies have reported reduced volume, disrupted intrinsic activity, and hypometabolism of hippocampus and hippocampal subregions in both $\mathrm{AD}$ and MCI patients [11, 12, 15, 17, 18]. However, there is still a lack of systematic examinations of the relationship between AD pathology in the hippocampal subregions and cognitive performance. More importantly, few have related intrinsic activity and metabolism based on the detailed subregional analyses on hippocampal. While hippocampus is widely recognized for its subregions with distinct functions [6], the localization of pathologies would indicate the specific roles of hippocampal subregions in symptomatology. Examining the relationship between intrinsic activity and metabolism within hippocampal subregions may also provide insight into disease pathogenesis.

Hybrid PET/MRI simultaneously evaluates resting-state brain structure, intrinsic activity, and glucose metabolism, which would provide optimal spatial and temporal registration of both modalities and clarify how neuronal function is impaired and contributes to the mechanisms underlying $\mathrm{AD}$ [19].
We hypothesized that different subregions have various contributions to the functionalities of hippocampus, and we aimed to investigate the aberrant of hippocampal subregions in MCI and $\mathrm{AD}$ patients regarding functional connectivity and metabolism using hybrid PET/MRI.

\section{Methods}

\section{Participants}

A total of 102 right-handed subjects were included in this study, comprising of 42 normal controls (NC), $38 \mathrm{MCI}$, and $22 \mathrm{AD}$ participants. Ethical approval was obtained from the Medical Research Ethics Committee of Xuanwu Hospital, Capital Medical University. Written informed consent was obtained from each participant and/or their legal representative before the PET/MR scan. Clinical diagnosis was established on the basis of a standard dementia screening that included medical history review, physical and neurological examinations, laboratory tests, neuropsychological tests, and brain ${ }^{18}$ F-FDG PET/MRI. Criteria for selecting the patients with AD met the National Institute of Neurological and Communicative Diseases and Stroke/Alzheimer's Disease and Related Disorders Association criteria for probable AD $[20,21]$. MCI was diagnosed according to the criteria of Petersen and colleagues [22]. All participants were assessed by neurologist with expertise in $\mathrm{AD}$ disorders. We excluded participants with Fazekas scores $>2$ to minimize the effect of vascular factors on functional connectivity [23], and other neurologic, psychiatric, or brain parenchyma diseases (e.g., stroke, tumors, and trauma) potentially related to cognitive impairment. The interval between neuropsychological assessments and simultaneous PET/MRI scan was within 30 days. To estimate cerebral microvascular impairment, the Fazekas scores were assessed by two experienced neuroradiologists [24].

\section{PET/MRI data acquisition}

Imaging data were collected with an integrated simultaneous time-of-flight (ToF) PET/MRI (Signa PET/MRI, GE Healthcare, WI, USA) by using a 19-channel head and neck union coil. Participants were scanned under resting conditions with eyes closed and dimmed light. The PET/MRI acquisition protocol was as same as our previous studies $[25,26]$. A sagittal 3D brain in volume (3D BRAVO) T1weighted sequence was used to acquire high-resolution anatomical images with the following parameters: repetition time $/$ echo time $=8.5 \mathrm{~ms} / 3.2 \mathrm{~ms}$, flip angle $=15^{\circ}$, voxel size $=1 \times 1 \times 1 \mathrm{~mm}^{3}$, and 188 slices. Ten-min fMRI and PET were acquired simultaneously. fMRI data were collected using a single-shot echo-planar imaging sequence: repetition time $/$ echo time $=2000 \mathrm{~ms} / 30 \mathrm{~ms}$, flip angle $=90^{\circ}$, gap $=$ 
$0.8 \mathrm{~mm}$, voxel size $=3.59 \times 3.59 \times 4.40 \mathrm{~mm}^{3}, 33$ slices covering the whole brain and 300 volumes with interleaved slice acquisitions. Both $\mathrm{T} 1$ and echo-planar imaging were generated with $\mathrm{B} 0$ calibration. PET emission data were acquired with 3D list mode. An 18-s 2-point Dixon scan was acquired for MRI-based PET attenuation correction. The decay- and attenuation-corrected PET images were reconstructed using an ordered subset expectation maximization algorithm $(8$ iterations, 32 subsets, and full width at half maximum of a Gaussian filter of $3.0 \mathrm{~mm}$ ) with ToF and point-spread function. The reconstructed PET image matrix was $192 \times 192$, the field of view was $350 \times 350 \mathrm{~mm}^{2}$, and the voxel size was $1.82 \times 1.82 \times 2.78 \mathrm{~mm}^{3}$, with spatial resolution of $4.5 \mathrm{~mm}[25-27]$.

\section{${ }^{18}$ F-FDG PET partial volume correction and quantification}

${ }^{18}$ F-FDG PET image spatial normalization and ROI ${ }^{18} \mathrm{~F}$-FDG SUVR calculation were described in our previous studies [28-30]. Briefly, all PET images were co-registered to matched T1-weighted 3D BRAVO MRI images. The structural MRI images were normalized to standard Montréal Neurological Institute (MNI) space using statistical parametric mapping software (SPM12, Wellcome Department of Imaging Neuroscience, London, United Kingdom) and CAT12 toolbox (http://dbm.neuro.uni-jena.de/cat/) with MRI template (image volume, $121 \times 145 \times 121$; voxel size, $1.5 \times 1.5 \times 1.5 \mathrm{~mm}^{3}$ in $\mathrm{x}, \mathrm{y}, \mathrm{z}$ ). The transformation parameters determined by MRI spatial normalization were then applied to the co-registered PET images for PET spatial normalization. To minimize partial volume effects on PET measurements, an iterative reblurred Van Cittert iteration method was applied to $10-\mathrm{min}{ }^{18} \mathrm{~F}$-FDG PET images for partial volume correction (PVC), where the PVC image $T_{k+1}(x)$ at $k$ th iteration for image $I(x)$ at voxel $x$ is updated by following equation.

$\mathrm{T}_{\mathrm{k}}+1(\mathrm{x})=\mathrm{T}_{\mathrm{k}}(\mathrm{x})+\alpha\left(\mathrm{h} *\left(\mathrm{I}-\mathrm{h} * \mathrm{~T}_{\mathrm{k}}\right)\right)(\mathrm{x})$

where $T_{0}(x)=I(x), h(x)$ is a 3D Gaussian kernel of 4.5-mm FWHM spatial smoothing function, step length $\alpha=1.5$, and the iteration was stopped if relative percent change of PVC images $<1 \%[28,30] .{ }^{18} \mathrm{~F}-\mathrm{FDG}$ SUVR images were calculated on the partial volume corrected PET images using cerebellum as reference tissue. The ROIs were then used to extract the SUVR on ${ }^{18} \mathrm{~F}$-FDG SUVR images.

\section{Structural MRI analysis}

The 3D high-resolution T1-weighted images data was processed using DPARSF voxel-based morphometry toolbox CAT12 based on SPM12 by adopting standard voxel- based morphometric (VBM) analysis processing routine. After normalizing all subjects to the standard MNI apace, segmenting (DARTEL) into gray matter, white matter, and cerebrospinal fluid images, the resulting images were modulated (without including affine component) and smoothed using a 4-mm FWHM 3D Gaussian filter. The gray matter volumes of total hippocampus and the hippocampal subregions were extracted based on the smoothed gray matter maps.

\section{Preprocessing of fMRI data}

The SPM12 and Data Processing Assistant for RestingState fMRI (DPARSF) toolbox (https://www.nitrc.org/ projects/dparsf/) [31] were used to perform fMRI data preprocessing. The first 10 functional volumes were removed to stabilize the fMRI signal and adapt the participants to the circumstances. The remaining 290 volumes were realigned to the middle volume for the head motion correction and then registered to the T1weighted sagittal images after slice timing correction. Nuisance regressors included the six motion vectors computed during the rigid body head motion correction. We regressed out 24 nuisance parameters (12 movement parameters and their first derivative). Eight participants (1 $\mathrm{NC}, 4 \mathrm{MCI}$, and $3 \mathrm{AD}$ ) were excluded based on excessive head motion (maximal translation $>3 \mathrm{~mm}$ or rotation $>3^{\circ}$ ). Then, the individual T1-weighted image was co-registered to the mean functional image using a linear transformation and segmented into gray matter, white matter and cerebrospinal fluid tissue probabilistic maps using the SPM12 unified segmentation algorithm. After normalizing to MNI standard template and smoothing with $\mathrm{FWHM}=4 \mathrm{~mm}$, the spatially normalized functional images were resampled to 3-mm isotropic voxels, and all voxel time courses were then temporally bandpass filtered with frequencies from 0.01 to $0.1 \mathrm{~Hz}$ to reduce the effect of low frequency drifts and high-frequency physiological noise.

\section{Seed-based functional connectivity analysis}

The masks of the region of interests (ROIs) (bilateral and unilateral hippocampus, CA1, CA2/3/DG, and subiculum) were extracted respectively from probabilistic cytoarchitectonic maps of the SPM Anatomy Toolbox for ROI-based functional connectivity analysis [32] (Fig. 1). After bandpass filtering all voxel time courses for frequencies from 0.01 to $0.1 \mathrm{~Hz}$, the voxel time courses of the ROIs were extracted, and then the functional connectivity of the hippocampal subregions was calculated within DPARSF. The individual mean time courses were extracted for bilateral and unilateral hippocampus, CA1, CA2/3/DG, and subiculum. Then 


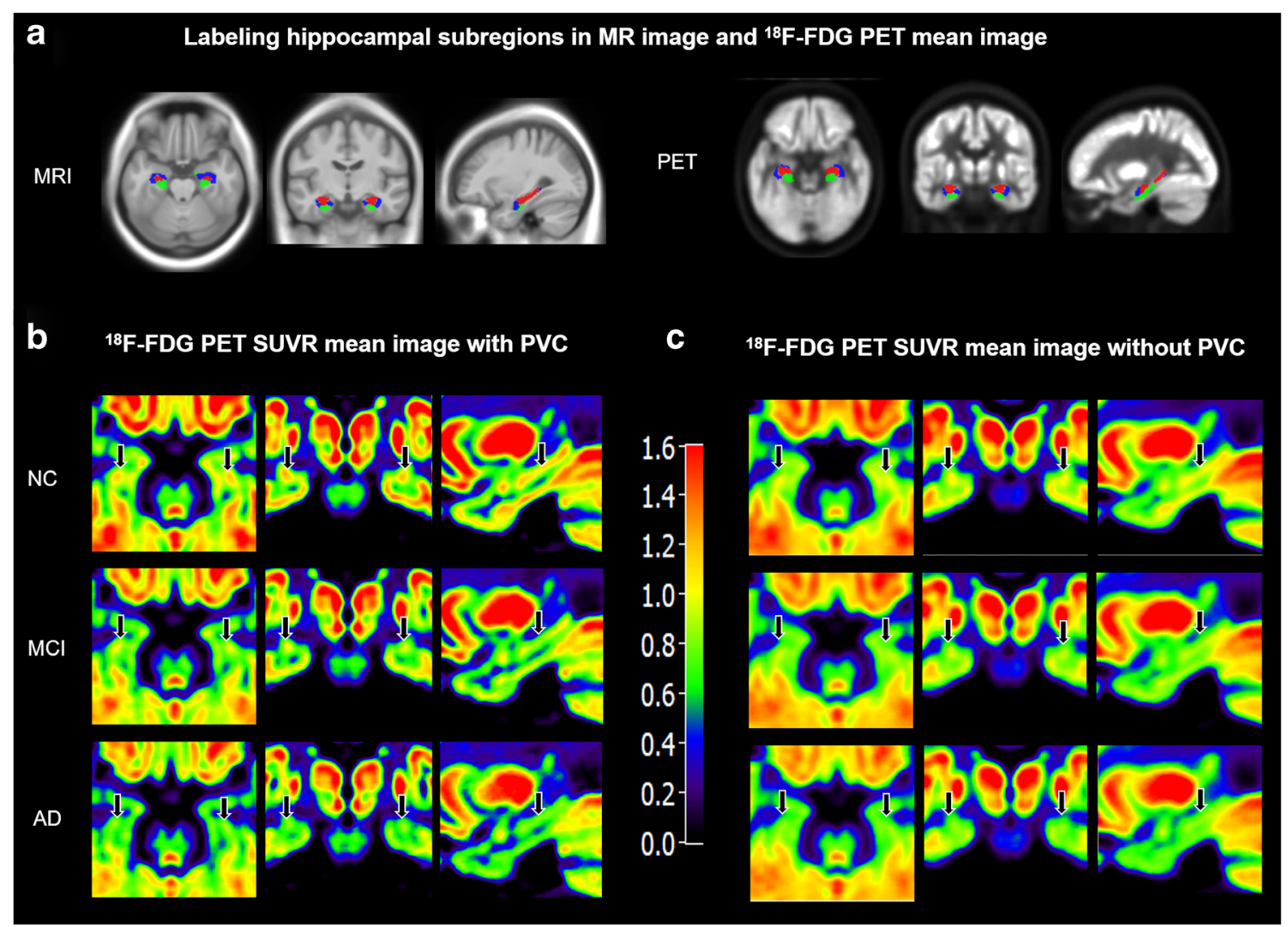

Fig. 1 Hippocampal subregions masks and mean ${ }^{18}$ F-FDG PET SUVR images with and without PVC. Hippocampal subregions masks overlaid on template MRI and PET in standard MNI space. Blue, CA1; red, CA2/ 3/DG; and green, subiculum. The mean SUVR images with PVC (b) illustrate increased contrast and spatial resolution as compared to the mean SUVR images without PVC (c). Note that the mean images are averaged over all participants in each group. The black arrows indicate the location of the hippocampus the individual ROI-based functional connectivity maps were computed for each region. Subsequently, we extracted and averaged the statistically significant ROI values from singlesubject functional connectivity maps after Fisher's z transformation.

\section{Statistical analysis}

Statistical Analysis System (SAS version 9.4, SAS Institute, Inc.) was used for all statistical analyses. Gender was compared with a chi-squared test. Each group comparison analysis in connectivity maps (obtained from the bilateral and unilateral hippocampus, CA1, CA2/3/DG, and subiculum region, respectively) was performed using two-sample $t$ tests. The significance levels of all clusters analyses were set to the voxel level at $P<0.01$ and the cluster level at $P<0.05$, after Gaussian random field correction. Age, gender, and education were used as covariates. A generalized linear model was used for group comparison on ROI volume and ${ }^{18} \mathrm{~F}$-FDG SUVR. A partial Pearson's correlations between ROIs connectivity, ${ }^{18} \mathrm{~F}$ FDG SUVR, and clinical assessments were calculated in all groups.

\section{Results}

\section{Demographics}

A total of $42 \mathrm{NC}, 38 \mathrm{MCI}$, and $22 \mathrm{AD}$ participants were studied. The demographic characteristics of all participants are listed in Table 1. Significant group differences were found in mini mental status examination (MMSE) scores, but not found $(P>0.05$ for all) in age, gender, and education. The AD $(P<0.001)$ and MCI $(P<0.001)$ group had significantly lower MMSE scores compared with NC.

\section{${ }^{18}$ F-FDG PET hypometabolism in hippocampal subregions}

The ${ }^{18}$ F-FDG SUVR mean images of hippocampus in Fig. 1 showed that the PVC remarkably improved spatial resolution and contrast. Therefore, the results here after presented in the study focused on the partial volume corrected SUVRs. Patient groups have significantly decreased ${ }^{18} \mathrm{~F}$-FDG PET metabolism compared to NC. In patients with $\mathrm{AD}$, significant reduction of ${ }^{18}$ F-FDG SUVR was observed within hippocampus, left hippocampus, right hippocampus, bilateral CA1, left 
Table 1 Demographics and neuropsychological assessments

\begin{tabular}{llllll}
\hline Parameter & NC & MCI & AD & $F$ value & $P$ value \\
\hline Total no. of participants & 42 & 38 & 22 & - & - \\
Women & 18 & 20 & 13 & 1.69 & 0.430 \\
Mean age in years (SD) & $65.81(6.14)$ & $68.89(8.46)$ & $67.68(7.17)$ & 1.81 & 0.169 \\
Mean education in years (SD) & $12.98(2.61)$ & $12.34(3.38)$ & $11.95(3.12)$ & 0.93 & 0.399 \\
Mean MMSE (SD) & $28.83(1.4)$ & $26.00(2.98)$ & $19.64(6.19)$ & 49.94 & $<0.001$ \\
CDR & 0 & 0.5 & $0.5-1$ & - & - \\
\hline
\end{tabular}

Group comparisons: generalized linear model (age, education, MMSE), gender (chi-square test)
CA1, right CA1, bilateral CA2/3/DG, left CA2/3/DG, right $\mathrm{CA} 2 / 3 / \mathrm{DG}$, bilateral subiculum, and left subiculum regions compared with those of NC. ${ }^{18}$ F-FDG SUVR within hippocampus, left hippocampus, right hippocampus, right $\mathrm{CA} 1$, bilateral CA2/3/DG, and left CA2/3/DG regions was significantly reduced in the $\mathrm{AD}$ patients compared with those of MCI. Compared with NC, MCI patients ${ }^{18}$ F-FDG SUVR was significantly reduced within hippocampus, left hippocampus, right hippocampus, right CA1, bilateral CA2/3/DG, left CA2/3/DG, right CA2/3/DG, bilateral subiculum, and left subiculum regions. However, only minor difference between $\mathrm{AD}$ and $\mathrm{MCI}$ patients was found in the left $\mathrm{CA} 1$, subiculum, left subiculum, and right subiculum regions (Fig. 2, Table 2).

\section{Functional connectivity in hippocampal subregions}

Group differences between NC and patient groups in functional connectivity were illustrated in Fig. 3 and Table 3. In AD patients, a significant decrease $(P<0.05)$ in functional connectivity, in comparison with MCI patients, was found between the following pairs of regions: (1) hippocampus and both the right superior frontal gyrus (SFG) and bilateral superior medial frontal gyrus (SMFG); (2) left hippocampus and both right SFG and bilateral SMFG; (3) right hippocampus and bilateral SMFG; (4) bilateral CA1 and all right SFG, bilateral SMFG, supplementary motor cortex (SMC), and middle frontal gyrus (MFG); (5) left CA1 and all bilateral SFG, SMFG, SMC, and right MFG; (6) right CA1 and all bilateral SFG, SMC, and SMFG. No difference was found within the CA2/3/DG and subiculum regions (Fig. 3a).

Compared with $\mathrm{NC}, \mathrm{AD}$ patients showed a significant decrease in functional connectivity between the following pairs of regions: (1) hippocampus and bilateral SMFG and SFG; (2) left hippocampus and bilateral SMFG; (3) right hippocampus and all bilateral SMFG, SFG, and right MFG; (4) bilateral CA1 and all bilateral SMFG, SFG, MFG, SMC, and
Fig. 2 Group differences in ${ }^{18} \mathrm{~F}$ FDG SUVR of hippocampus and hippocampal subregions. Bar graphs display the mean (standard deviation) of the age-, gender-, and education-corrected ${ }^{18} \mathrm{~F}-\mathrm{FDG}$ SUVR. Mean ${ }^{18}$ F-FDG SUVR reductions in patients with $\mathrm{AD}$ and MCI compared with $(P<0.05) \mathrm{NC}$ in hippocampus (a), CA1 (b), CA2/3/DG (c), and subiculum (d). Red, AD; blue, $\mathrm{MCI}$; and green, NC. $P$ value as defined using a generalized linear model to detect significant difference in the three groups. $* P<0.05, * * P<0.01$ $* * * P<0.001$

\section{a Hippocampus}

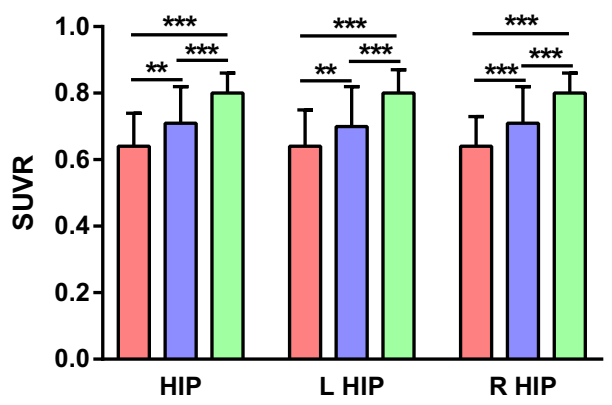

C CA2/3/DG

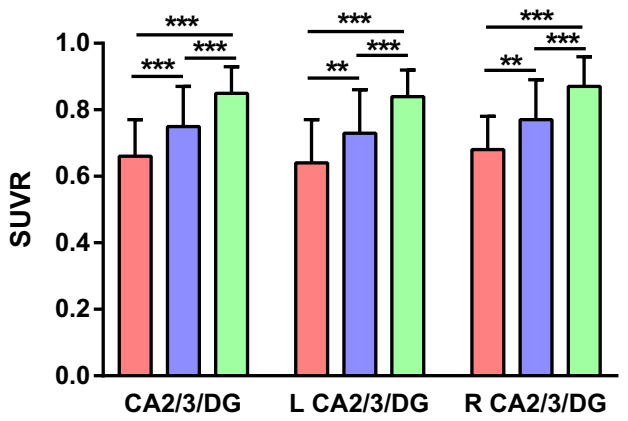

\section{b CA1}

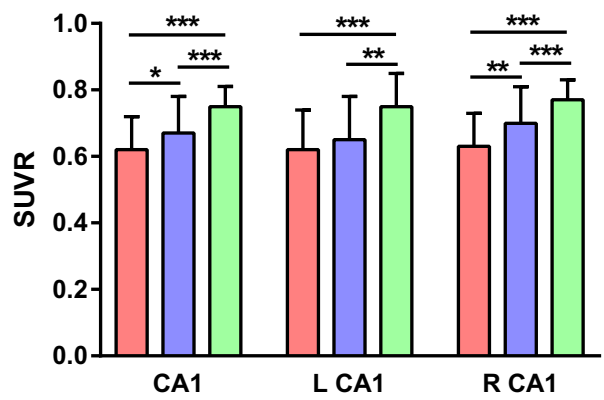

d Subiculum

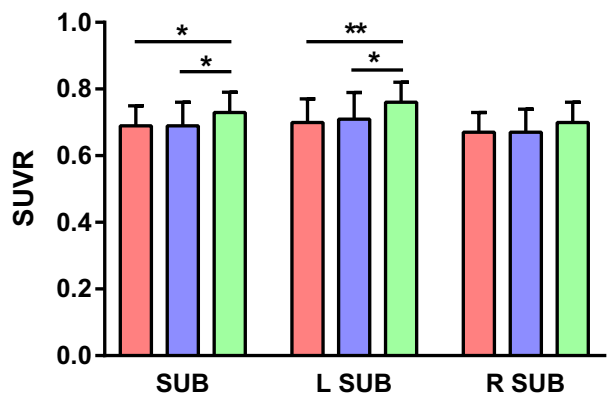


Table 2 Averaged ${ }^{18}$ F-FDG SUVR in hippocampal subregions

\begin{tabular}{lllllr}
\hline Regions & AD & MCI & NC & $F$ value & $P$ value \\
\hline Hippocampus & $0.64 \pm 0.10^{\mathrm{bc}}$ & $0.71 \pm 0.11^{\mathrm{b}}$ & $0.80 \pm 0.06$ & 24.59 & $<0.001$ \\
L Hippocampus & $0.64 \pm 0.11^{\mathrm{bd}}$ & $0.70 \pm 0.12^{\mathrm{b}}$ & $0.80 \pm 0.07$ & 19.33 & $<0.001$ \\
R Hippocampus & $0.64 \pm 0.09^{\mathrm{bd}}$ & $0.71 \pm 0.11^{\mathrm{b}}$ & $0.79 \pm 0.06$ & 18.49 & $<0.001$ \\
CA1 & $0.62 \pm 0.10^{\mathrm{bc}}$ & $0.67 \pm 0.11^{\mathrm{d}}$ & $0.75 \pm 0.06$ & 16.45 & $<0.001$ \\
L CA1 & $0.62 \pm 0.12^{\mathrm{b}}$ & $0.65 \pm 0.13^{\mathrm{b}}$ & $0.75 \pm 0.10$ & 13.13 & $<0.001$ \\
R CA1 & $0.63 \pm 0.10^{\mathrm{bc}}$ & $0.70 \pm 0.11^{\mathrm{b}}$ & $0.77 \pm 0.06$ & 16.24 & $<0.001$ \\
CA2/3/DG & $0.66 \pm 0.11^{\mathrm{bd}}$ & $0.75 \pm 0.12^{\mathrm{b}}$ & $0.85 \pm 0.08$ & 22.90 & $<0.001$ \\
L CA2/3/DG & $0.64 \pm 0.13^{\mathrm{bd}}$ & $0.73 \pm 0.13^{\mathrm{b}}$ & $0.84 \pm 0.08$ & 21.72 & $<0.001$ \\
R CA2/3/DG & $0.68 \pm 0.10^{\mathrm{bd}}$ & $0.77 \pm 0.12^{\mathrm{b}}$ & $0.87 \pm 0.09$ & 19.28 & $<0.001$ \\
Subiculum & $0.69 \pm 0.06^{\mathrm{b}}$ & $0.69 \pm 0.07^{\mathrm{a}}$ & $0.73 \pm 0.06$ & 6.11 & 0.003 \\
L Subiculum & $0.70 \pm 0.07^{\mathrm{b}}$ & $0.71 \pm 0.08^{\mathrm{b}}$ & $0.76 \pm 0.06$ & 7.69 & 0.001 \\
R Subiculum & $0.67 \pm 0.06^{\mathrm{a}}$ & $0.67 \pm 0.07^{\mathrm{a}}$ & $0.70 \pm 0.06$ & 3.95 & 0.022 \\
\hline
\end{tabular}

a compared with $\mathrm{NC}, P<0.05$

${ }^{\mathrm{b}}$ compared with NC, $P<0.01$

${ }^{\mathrm{c}}$ compared with $\mathrm{MCI}, P<0.05$

d compared with MCI, $P<0.01$ precuneus; (5) left CA1 and all bilateral SMFG, MFG, and precuneus; (6) right CA1 and all bilateral SMFG, SFG, MFG, and precuneus; (7) bilateral $\mathrm{CA} 2 / 3 / \mathrm{DG}$ and all bilateral SMFG, left CA2/3/DG, and right SMFG; (8) right CA2/3/ DG and right SFG; (9) bilateral subiculum and right $\mathrm{SFG}$; and (10) right subiculum and SFG $(P<0.05)$. No difference was found within the left subiculum region (Fig. 3b).

In MCI patients, a significant decrease in functional connectivity, in comparison with NC $(P<0.05)$, was found between the following pairs of regions: (1) hippocampus and both left MFG and SFG; (2) right hippocampus and left precuneus; (3) bilateral CA1 and left inferior frontal gyrus (IFG); (4) right CA1 and both left IFG and right precuneus; (5) CA2/3/DG and both right inferior occipital gyrus and lingual; (6) right CA2/3/DG and all left SFG, right SFG, and bilateral MFG; (7) subiculum and all bilateral middle cingulate cortex, PCC, middle occipital gyrus (MOG), superior occipital gyrus, and cuneus; (8) left subiculum and all bilateral middle cingulate, precuneus, right MOG, angular, and cuneus; and (9) right subiculum and all right MOG, cuneus, and bilateral precuneus. No difference existed within the left hippocampus, CA1, and CA2/3/DG regions (Fig. 3c).

\section{Association between functional connectivity and MMSE}

The MMSE score predicted a higher right CA1 region functional connectivity with the right precuneus in patients with MCI $(P=0.010, r=0.415$; Fig. $4 \mathrm{a})$ while a higher right subiculum region functional connectivity with the right $\mathrm{SFG}$ in patients with $\mathrm{AD}(P=0.044, r=0.434$; Fig. $4 \mathrm{~b})$. There was neither association between MMSE and other hippocampal subregions in functional connectivity nor between MMSE and all the hippocampal subregions in ${ }^{18}$ F-FDG SUVR (all $P>0.05)$.

\section{Association among functional connectivity, ${ }^{18}$ F-FDG SUVR, and volume in hippocampal subregions}

Partial Pearson's correlation analysis demonstrated that the decreased left CA2/3DG-SMFG connectivity in AD compared to $\mathrm{NC}$ group was associated with left CA2/3DG higher ${ }^{18} \mathrm{~F}-\mathrm{FDG}$ SUVR $(P=0.010, r=-0.538$; Fig. 5 a, b) and volume $(P=$ $0.035, r=-0.487$; Fig. $5 \mathrm{c}$ ). There was a linear correlation between the left CA2/3DG volume and ${ }^{18} \mathrm{~F}$-FDG SUVR in AD patients $(P<0.001, r=0.737$; Fig. 5 d $)$. No correlation was found between functional connectivity and metabolism in other hippocampal subregions (all $P>0.05$ ). The volumes of the hippocampal subregions are shown in Fig. 6.

\section{Discussion}

To the best of our knowledge, this is the first study to evaluate subregional hippocampal resting-state brain functional connectivity and glucose metabolism in AD study using simultaneous PET/fMRI. Hybrid PET/MRI is capable of simultaneous evaluating resting-state intrinsic activity, glucose metabolism, and gray matter volume, which could provide evidence for better understanding the neurodegenerative mechanisms underlying AD. Our hippocampal subregion-based analysis on simultaneous ${ }^{18} \mathrm{~F}$-FDG PET/fMRI demonstrated that patient groups had significantly reduced functional connectivity and ${ }^{18} \mathrm{~F}$-FDG SUVR in comparison with $\mathrm{NC}$ in most 
a

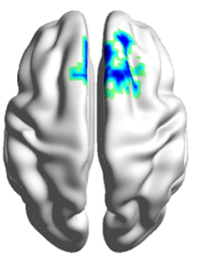

HIP

b

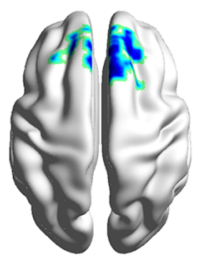

HIP

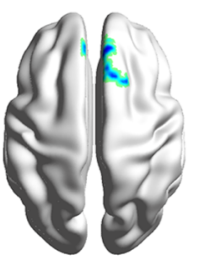

CA2/3/DG

C

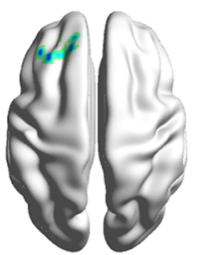

HIP

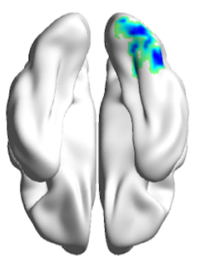

CA2/3/DG

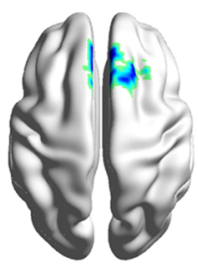

HIPL

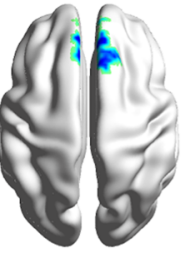

HIPL

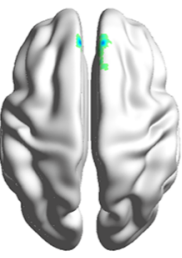

CA2/3/DGL

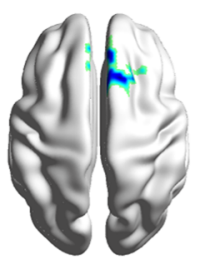

HIPR

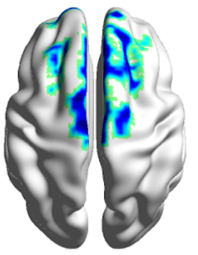

CA1

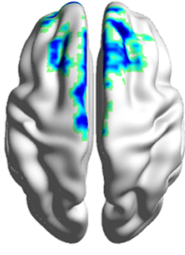

CA1L

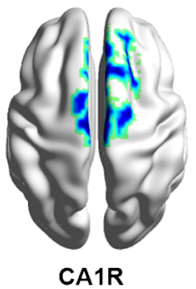

$-4.09$

AD $<$ NC

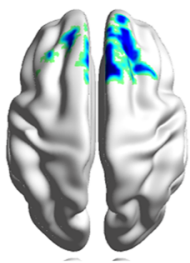

HIPR

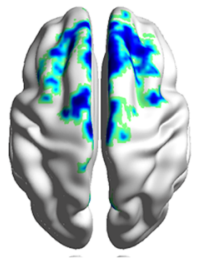

CA1

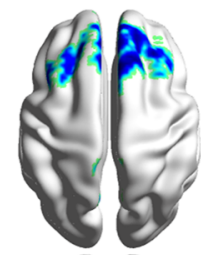

CA1L
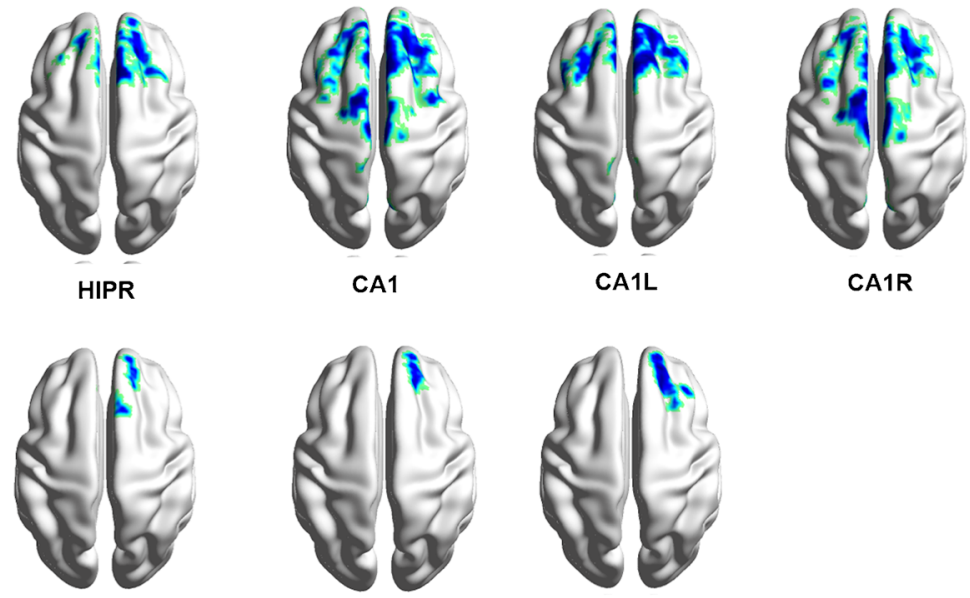

CA2/3/DGR

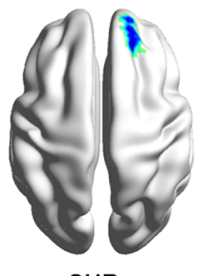

SUB

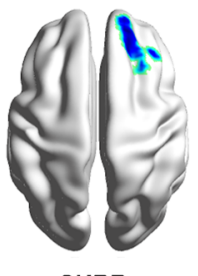

SUBR

$\mathrm{MCl}<\mathrm{NC}$

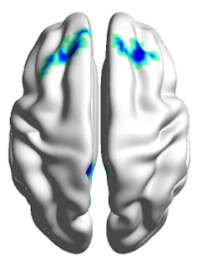

HIPR

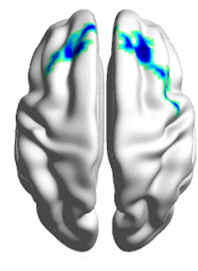

CA2/3/DGR

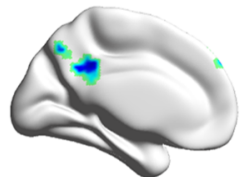

HIPR

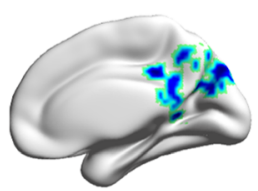

SUB

Fig. 3 Group differences in hippocampal subregional functional connectivity. The results were mapped on the brain surface using BrainNet Viewer [48] showing reduced hippocampal subregional

of the hippocampal subregions. Specifically, we found a negative correlation between the decreased left CA2/3DG-SMFG connectivity and local ${ }^{18} \mathrm{~F}$-FDG PET hypometabolism in AD patients. In addition, we observed that the right CA1precuneus connectivity was associated with cognitive impairment in participants with MCI.

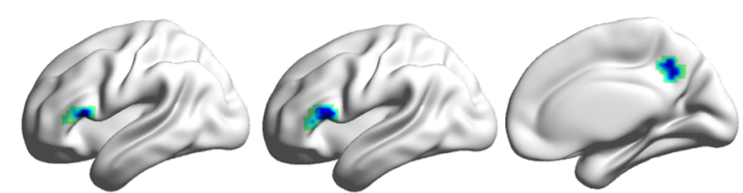

CA1

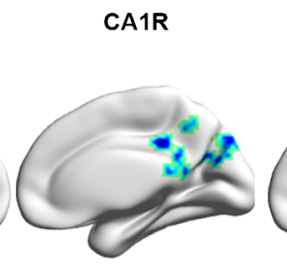

CA1R

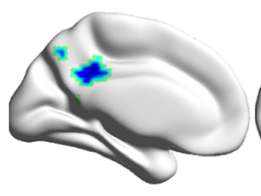

SUBL

SUBL

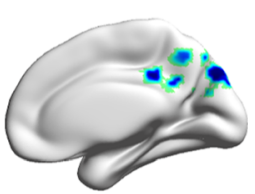

SUBR

functional connectivity in $\mathrm{AD}$ compared with $\mathrm{MCI}$ (a) or $\mathrm{NC}$ (b), $\mathrm{MCI}$ compared with NC (c) (two-sample $t$ test, voxel level $P<0.01$, cluster level $P<0.05$, Gaussian random field corrected). Colors indicate t scores

Our inter-group subregion-based analysis on fMRI showed that all hippocampal subregions in both patient groups $(\mathrm{AD}$ and $\mathrm{MCI}$ ) had decreased functional connectivity comparing to $\mathrm{NC}$, and CA1 subregion could potentially serve as a major imaging indicator differentiating $\mathrm{AD}$ and $\mathrm{MCI}$ patients. A recently published study analyzed specific functional connectivity of CA1, 
Table 3 Group differences in subregional hippocampal functional connectivity

\begin{tabular}{|c|c|c|c|c|}
\hline Seed regions & Cluster & Cluster size & $T$ value & $\mathrm{MNI}(\mathrm{x}, \mathrm{y}, \mathrm{z})$ \\
\hline \multicolumn{5}{|l|}{$\mathrm{AD}<\mathrm{MCI}$} \\
\hline Hippocampus & R SFG \& L/R SMFG & 273 & -4.512 & $15,18,60$ \\
\hline L Hippocampus & R SFG \& L/R SMFG & 169 & -4.087 & $12,21,57$ \\
\hline R Hippocampus & L/R SMFG & 152 & -4.523 & $6,36,54$ \\
\hline CA1 & L/R SFG, SMFG, SMC \& MFG & 1190 & -4.958 & $-6,-15,72$ \\
\hline L CA1 & L/R SFG, SMFG, SMC \& R MFG & 958 & -4.847 & $-6,30,51$ \\
\hline R CA1 & L/R SFG, SMC \& SMFG & 339 & -4.516 & $6,36,54$ \\
\hline \multicolumn{5}{|l|}{$\mathrm{AD}<\mathrm{NC}$} \\
\hline Hippocampus & L/R SMFG \& SFG & 476 & -4.759 & $6,36,57$ \\
\hline L Hippocampus & L/R SMFG & 209 & -4.396 & $6,42,51$ \\
\hline R Hippocampus & L/R SMFG, SFG \& R MFG & 488 & -5.069 & $9,27,60$ \\
\hline \multirow[t]{2}{*}{ CA1 } & L/R SMFG, SFG, MFG \& SMC & 1286 & -5.620 & $6,27,57$ \\
\hline & L/R Precuneus & 152 & -4.178 & $-3,-57,72$ \\
\hline \multirow[t]{2}{*}{ L CA1 } & L/R SMFG, SFG & 987 & -5.069 & $6,27,57$ \\
\hline & L/R Precuneus & 131 & -4.534 & $-6,-57,72$ \\
\hline \multirow[t]{2}{*}{ R CA1 } & L/R SMFG, SFG \& MFG & 820 & -5.412 & $6,27,57$ \\
\hline & L/R Precuneus & 115 & -3.949 & $0,-69,51$ \\
\hline $\mathrm{CA} 2 / 3 / \mathrm{DG}$ & L/R SMFG & 109 & -4.190 & $6,36,57$ \\
\hline $\mathrm{L} \mathrm{CA} 2 / 3 / \mathrm{DG}$ & R SMFG & 62 & -3.497 & $9,42,51$ \\
\hline R CA2/3/DG & R SFG & 50 & -3.495 & $15,42,39$ \\
\hline Subiculum & R SFG & 57 & -3.821 & $21,42,39$ \\
\hline R Subiculum & R SFG & 153 & -4.205 & $21,51,33$ \\
\hline \multicolumn{5}{|l|}{$\mathrm{MCI}<\mathrm{NC}$} \\
\hline Hippocampus & L MFG \& SFG & 64 & -3.701 & $-36,36,39$ \\
\hline R Hippocampus & L Precuneus & 93 & -3.533 & $-9,-42,33$ \\
\hline CA1 & L IFG & 71 & -3.959 & $-36,3,18$ \\
\hline \multirow[t]{2}{*}{ R CA1 } & L IFG & 64 & -3.832 & $-48,27,9$ \\
\hline & R Precuneus & 75 & -3.547 & $12,-60,39$ \\
\hline $\mathrm{CA} 2 / 3 / \mathrm{DG}$ & R Inferior Occipital gyrus \& Lingual & 151 & -3.695 & $21,-87,-6$ \\
\hline $\mathrm{R} \mathrm{CA} 2 / 3 / \mathrm{DG}$ & L/R SFG \& L/R MFG & 169 & -4.406 & $-27,42,39$ \\
\hline \multirow[t]{2}{*}{ Subiculum } & L/R Middle Cingulate \& PCC & 424 & -4.335 & $6,-36,36$ \\
\hline & R MOG, Superior Occipital gyrus \& Cuneus & 489 & -4.654 & $9,-84,39$ \\
\hline \multirow[t]{2}{*}{ L Subiculum } & L/R Middle Cingulate \& Precuneus & 249 & -4.175 & $6,-36,36$ \\
\hline & R MOG, Angular \& Cuneus & 311 & -4.231 & $39,-72,27$ \\
\hline \multirow[t]{2}{*}{ R Subiculum } & R MOG \& Cuneus & 225 & -5.256 & $12,-81,39$ \\
\hline & L/R Precuneus & 173 & -4.583 & $6,-36,36$ \\
\hline
\end{tabular}

Voxel level $P<0.01$ and the cluster level $P<0.05$, after Gaussian random field correction

subiculum, and CA2/3/4/DG using healthy elderly and their changes in patients with amnestic MCI [12]. The authors found a significant reduced connectivity within the subiculum network (with frontal cortex and PCC) in amnestic MCI patients. Our current findings are partly in line with this result, showing that the individuals with MCI had reduced connectivity between subiculum and PCC, right MOG, superior occipital gyrus, and cuneus. Besides, our study also demonstrated that the hippocampus, CA1, and CA2/3/DG had reduced connectivity in patient groups compared with $\mathrm{NC}$, mainly in the frontal cortex including SMFG, SFG, precuneus, PCC, etc. Using the whole hippocampus as a seed, we showed that the right whole hippocampus-medial prefrontal cortex, cingulate cortex, right cuneus extending into precuneus, left cuneus, and PCC functional connectivity were disrupted [18]. More specifically, as shown in Fig. 3a, CA1 was the only subregion exhibiting group differences between $\mathrm{AD}$ and $\mathrm{MCI}$ patients. The functional connectivity of CA1 decreased in patients with $\mathrm{AD}$ mainly in the frontal cortex and precuneus areas, which are the hubs of the default mode network. These results concurred well with a 
Fig. 4 Correlations between hippocampal subregional functional connectivity and MMSE. a Decreased right CA1precuneus functional connectivity in patients with $\mathrm{MCI}$ was positively correlated with MMSE. b Decreased right subiculumsuperior frontal gyrus functional connectivity in patients with $\mathrm{AD}$ was positively correlated with MMSE. Fitted lines, $P$ values, and 95\% confidence intervals are displayed from linear regression models

\section{a $\mathrm{MCl}$}

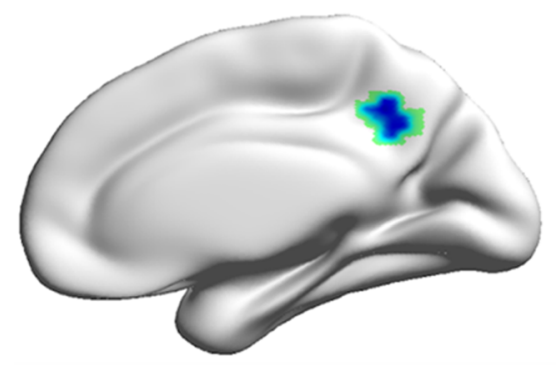

FC:CA1_R-Precuneus

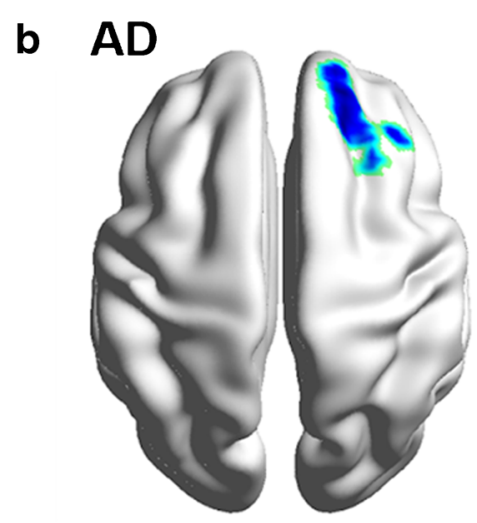

FC: SUB_R-SFG
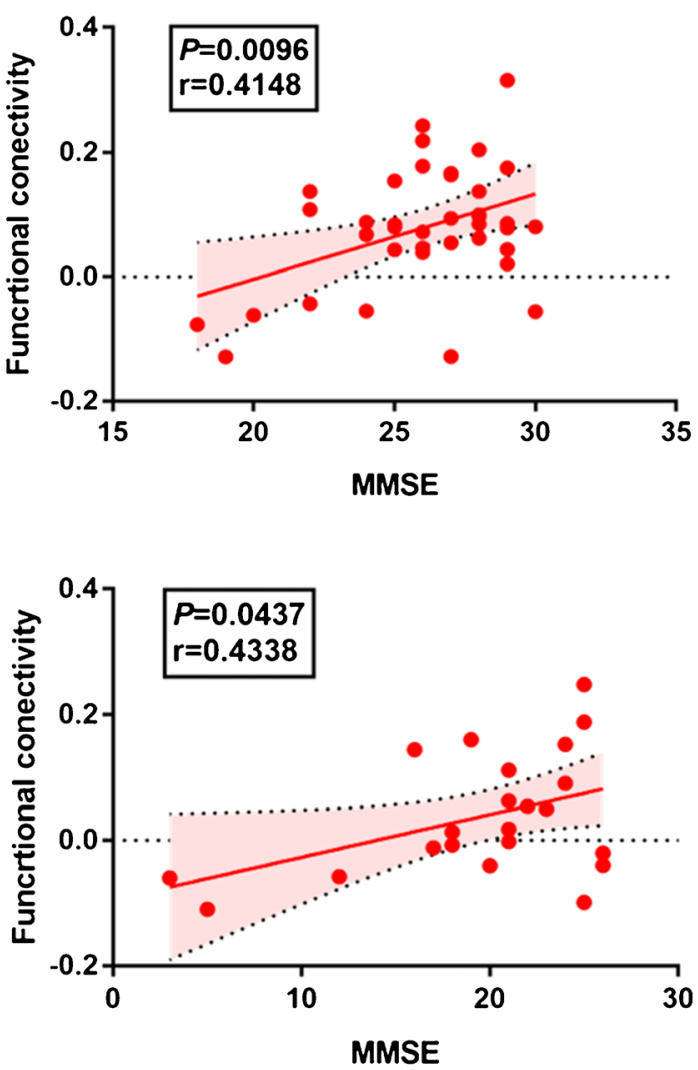

neuroimaging study that showed reduced hippocampal functional connectivity in $\mathrm{AD}$ [33] and also confirmed our earlier study of resting-state fMRI functional connectivity using the total hippocampus as a seed [34].

Our hippocampal subregional analysis on ${ }^{18} \mathrm{~F}$-FDG PET metabolism showed that both patient groups possessed reduced SUVR in hippocampal subregions ( $\mathrm{AD}<\mathrm{MCI}<\mathrm{NC})$, and the decreased left CA2/3/DG-SMFG connectivity in AD compared to NC group exhibited a negative correlation with the left $\mathrm{CA} 2 / 3 / \mathrm{DG}{ }^{18} \mathrm{~F}-\mathrm{FDG}$ PET metabolism in AD patients. A recent study of Choi et al. focused on comparing the glucose metabolism of hippocampal subregions in mildAD patients and healthy controls [15]. Their results revealed that the reductions in metabolic activity were found varying along the hippocampal axis in early-stage AD patients. When considering the hippocampal body as an entire structure, there was significantly lower glucose metabolism in the $\mathrm{AD}$ group than that of control group just in the left CA2/3 and CA4/DG. Apart from the subregions specified by Choi et al., our results showed that all of the hippocampal subregions had reduced metabolism except for the right subiculum, which was supported by our volume results. The subiculum gray matter volume reduction in participants with MCI and AD is not consistent in the previous studies [35-37]. Although we found a trend of subiculum gray matter volume reduction in participants with $\mathrm{MCI}$ and $\mathrm{AD}$ as compared to healthy controls $(P=0.5$ and $P=0.1$, respectively) which is consistent with previous amnestic MCI study [37], the reduction of subiculum volume still required further investigation and validation. The novelty of our current study is the relationship between metabolism and strength of hippocampal subregions functional connectivity to remote brain regions in $\mathrm{AD}$ patients, which supports the hippocampus disconnection hypothesis, i.e., uncoupling of hippocampus from cortical inputs system may contribute to disinhibition like changes of intrahippocampal activity [38, 39]. As shown in Fig. 5b, the association between left CA2/ 3/DG-SMFG connectivity and ${ }^{18}$ F-FDG PET metabolism revealed a negative correlation and was specific for $\mathrm{AD}$ patients, without the presence in neither MCI patients nor NC participants. Contrary to a previous finding suggesting no relationship between functional connectivity and ${ }^{18} \mathrm{~F}$ FDG PET metabolism in the posterior default mode network in the AD group [40], our results supported a recent evidence from a task fMRI study in humans indicating that greater hippocampal activation in amnestic MCI localized in the CA3/DG region [37], suggesting similar neural dysfunction. A therapeutic study in amnestic MCI showed that levetiracetam could reduce CA3/DG activation and improve cognitive function [41], indicating that targeting excess hippocampal activity has therapeutic potential. Overall, by integrating ${ }^{18}$ F-FDG PET and fMRI data, we found a robust 
a FC: CA2/3/DG_L-SMFG

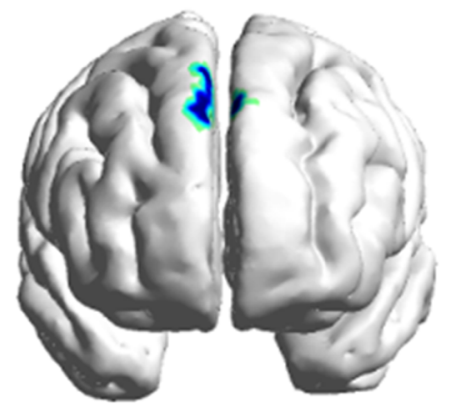

c CA2/3/DG_L

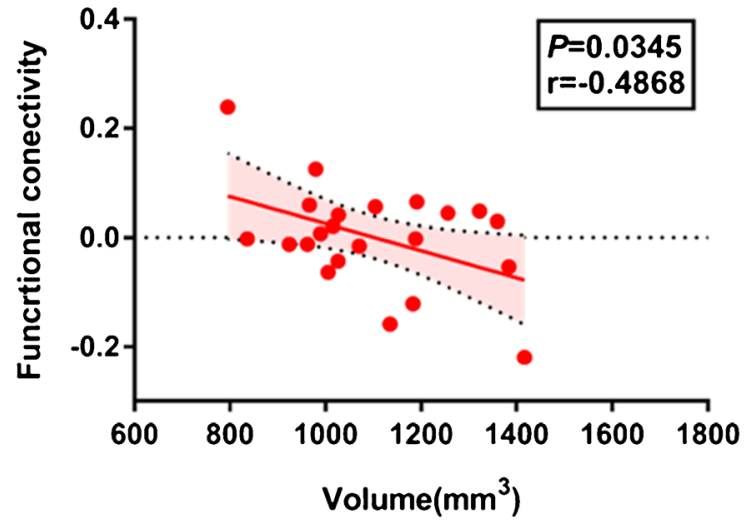

Fig. 5 Association among left CA2/3/DG region functional connectivity, ${ }^{18}$ F-FDG SUVR, and volume in AD subjects. Decreased left CA2/3DG$\mathrm{SMFG}$ connectivity in $\mathrm{AD}$ compared to $\mathrm{NC}$ group (a) was associated with b CA2/3/DG_L

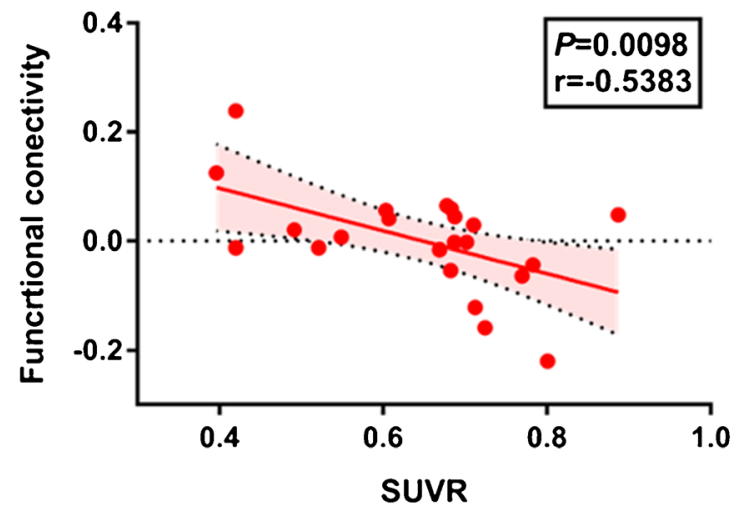

d CA2/3/DG_L

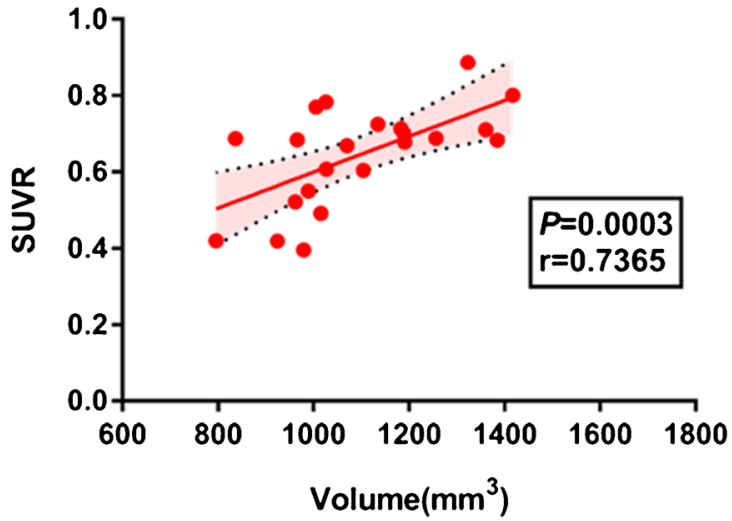

local ${ }^{18} \mathrm{~F}$-FDG SUVR (b) and volume (c). The left CA2/3DG volume in $\mathrm{AD}$ was associated with local ${ }^{18} \mathrm{~F}$-FDG SUVR (d). Fitted lines, $P$ values, and $95 \%$ confidence intervals are displayed from linear regression models
Fig. 6 Group differences in volumes of hippocampus and hippocampal subregions (mean \pm standard deviation, $\mathrm{mm}^{3}$ ). Generalized linear model followed by least square means post hoc analysis showed that the volume reduced in patients with $\mathrm{AD}$ and $\mathrm{MCI}$ compared with $\mathrm{NC}$ in hippocampus (a), CA1 (b), CA2/3/DG (c), and subiculum (d) with the age-, gender-, and education-corrected $(P<0.05)$. Red, AD; blue, MCI; and green, NC. $* P<0.05, * * P<0.01$, $* * * P<0.001$
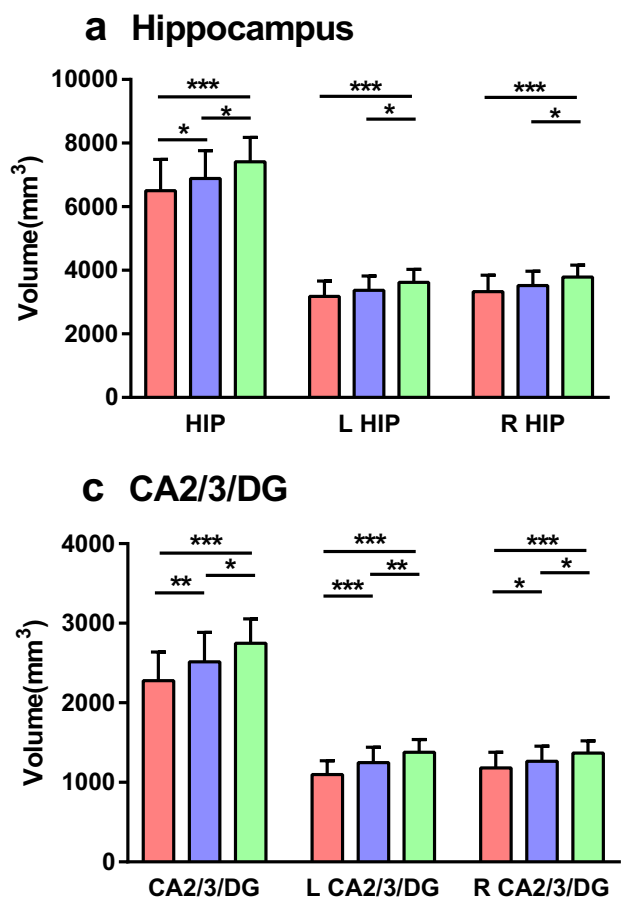

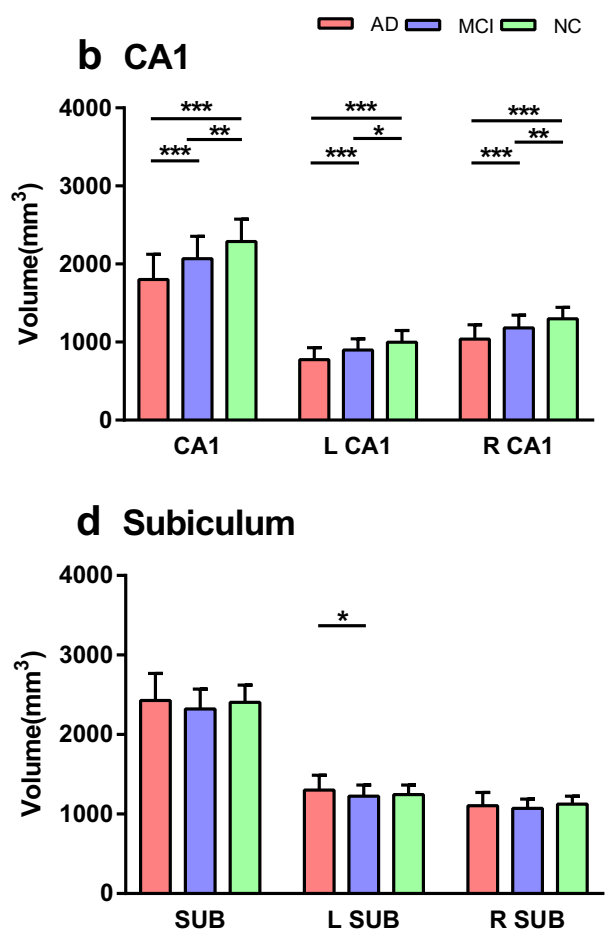


Table 4 Statistical significance of group difference in subregional hippocampal ${ }^{18}$ F-FDG SUVRs

\begin{tabular}{|c|c|c|c|c|c|c|}
\hline \multirow[t]{2}{*}{ Regions } & \multicolumn{2}{|c|}{$\begin{array}{l}\mathrm{AD} \text { vs } \mathrm{MCI} \\
P \text { value }\end{array}$} & \multicolumn{2}{|c|}{$\begin{array}{l}\mathrm{AD} \text { vs } \mathrm{NC} \\
P \text { value }\end{array}$} & \multicolumn{2}{|c|}{$\begin{array}{l}\text { MCI vs NC } \\
P \text { value }\end{array}$} \\
\hline & PVC & Non-PVC & PVC & Non-PVC & PVC & Non-PVC \\
\hline Hippocampus & 0.002 & 0.010 & $<0.001$ & $<0.001$ & $<0.001$ & $<0.001$ \\
\hline L Hippocampus & 0.010 & 0.023 & $<0.001$ & $<0.001$ & $<0.001$ & $<0.001$ \\
\hline R Hippocampus & $<0.001$ & 0.008 & $<0.001$ & $<0.001$ & $<0.001$ & $<0.001$ \\
\hline CA1 & 0.016 & 0.028 & $<0.001$ & $<0.001$ & $<0.001$ & $<0.001$ \\
\hline L CA1 & 0.171 & 0.110 & $<0.001$ & $<0.001$ & 0.002 & $<0.001$ \\
\hline R CA1 & 0.002 & 0.011 & $<0.001$ & $<0.001$ & $<0.001$ & $<0.001$ \\
\hline $\mathrm{CA} 2 / 3 / \mathrm{DG}$ & $<0.001$ & 0.005 & $<0.001$ & $<0.001$ & $<0.001$ & $<0.001$ \\
\hline $\mathrm{L} \mathrm{CA} 2 / 3 / \mathrm{DG}$ & 0.003 & 0.008 & $<0.001$ & $<0.001$ & $<0.001$ & $<0.001$ \\
\hline $\mathrm{R} \mathrm{CA} 2 / 3 / \mathrm{DG}$ & $<0.001$ & 0.007 & $<0.001$ & $<0.001$ & $<0.001$ & $<0.001$ \\
\hline Subiculum & 0.598 & 0.345 & 0.018 & 0.002 & 0.032 & 0.010 \\
\hline L Subiculum & 0.483 & 0.246 & 0.004 & $<0.001$ & 0.012 & 0.005 \\
\hline R Subiculum & 0.971 & 0.575 & 0.1765 & 0.065 & 0.126 & 0.093 \\
\hline
\end{tabular}

A generalized linear model including covariates age, gender, and education level was used to detect ${ }^{18} \mathrm{~F}-\mathrm{FDG}$ ROI SUVR significant difference among NC, MCI, and AD groups. The $P$ values were reduced (italic) for ROI SUVRs calculated from PET with PVC relationship between left CA2/3/DG-SMFG connectivity and local ${ }^{18}$ F-FDG SUVR in AD patients.

As for the correlation analysis of hippocampal subregions and MMSE, we found that the right CA1-precuneus and right subiculum-SMFG connectivity were positively correlated with cognitive impairment in MCI and AD patients, respectively. A previous animal research study showing that the pattern of CA1 neuron activation indicated cognitive features [42]. In patients with $A D$, the CA1 region was severely affected by neuron number and neurofibrillary tangle, which were significantly related to CDR scores [43]. A recent autopsy study also found that the degree of Lewy body pathology in CA1, but not CA2, predicted pre-mortem episodic memory impairment in patients with Lewy body dementia and Parkinson's disease [5,44]. Their findings suggested that CA1 might be more functionally relevant than CA2 and subiculum regions in memory impairment. These results supported the view that excess hippocampal activation directly contributed to the cognitive decline in prodromal $\mathrm{AD}[41,45]$.

The partial volume effects on the subregional hippocampal neurodegeneration measurements by PET were minimized by voxelwise PVC. As demonstrated by Fig. 1 and Table 4, the statistical significance levels were improved by PET with PVC in testing the ROI FDG SUVR differences between $\mathrm{AD}$ and $\mathrm{MCI}$ groups in left and right hippocampus, CA2/3/ DG, and right CA1. This is consistent with our previous Alzheimer's Disease Neuroimaging Initiative studies in small ROIs including amygdala and entorhinal cortex, where the PET spatial resolution of 8-mm FWHM $[28,29]$. In the study, we also performed RBV (region-based voxelwise)-RVC (reblurred Van Cittert iteration) PVC algorithm [46]. The statistical analysis based on PET with RBV-RVC PVC did not change any conclusions from RVC-based analysis (not shown). Note that the subregional hippocampal neurodegeneration measurements by ${ }^{18} \mathrm{~F}$-FDG PET are consistent with the ones from high-resolution T1-weighted MRI (Figs. 2, 5, and 6). We also confirmed that the subregional hippocampal functional activities from the spatially smoothed fMRI (Gaussian 3D smooth filter with FWHM $=4 \mathrm{~mm}$, see "Method" section) are separable with 3-T MRI scanners [16]. We realized that the biological $\mathrm{AD}$ definition was recently proposed by the National Institute on Aging and Alzheimer's Association for the $\mathrm{A}(\mathrm{A} \beta)-\mathrm{T}(\mathrm{tau})-\mathrm{N}$ (neuro-degeneration) research framework [47]. We will have AT(N) CSF or imaging measurements in future study to minimize AD pathology biases.

In summary, the subregional hippocampal level analysis revealed hypometabolism, lower gray matter volume, aberrant functional connectivity, and their relationship in $\mathrm{MCI}$ and $\mathrm{AD}$ patients. In addition, the right CA1-precauneus connectivity was related to the cognition in MCI patients, and the left CA2/ 3/DG functional connectivity was correlated to hypometabolism in AD patients. Our findings demonstrate that the associations existed at subregional hippocampal level between the functional connectivity and neurodegeneration measured by simultaneous PET/MRI.

Acknowledgments The authors thank Hongwei Yang, Jie Ma, and Dongmei Shuai for the assistance with the patient studies and Zhongwei Chen for the data analysis.

Author contributions All authors contributed to the study conception and design. Study was designed by Jie Lu, Yun Zhou, and Zhigang Qi. Material preparation, data collection and analysis were performed by 
Shaozhen Yan, Bixiao Cui, Zhilian Zhao, Yanhong An, Liyan Qiao and Ying Han. The first draft of the manuscript was written by Shaozhen Yan, Chaojie Zheng and all authors commented on previous versions of the manuscript. All authors read and approved the final manuscript.

Funding information This study was supported by the Beijing Municipal Administration of Hospitals' Ascent Plan (No. DFL20180802), the National Key Research and Development Program of China (Grant No. 2016YFC0103909), a special fund from the Key Laboratory of Neurodegenerative Diseases, Ministry of Education (PXM2019 026283 000003), the Beijing Municipal Commission of Science and Technology (Z171100000117001), and the National Natural Science Foundation of China (Grant No. 81801677, 81141018).

\section{Compliance with ethical standards}

Competing interests The authors declare that they have no competing interest.

Open Access This article is licensed under a Creative Commons Attribution 4.0 International License, which permits use, sharing, adaptation, distribution and reproduction in any medium or format, as long as you give appropriate credit to the original author(s) and the source, provide a link to the Creative Commons licence, and indicate if changes were made. The images or other third party material in this article are included in the article's Creative Commons licence, unless indicated otherwise in a credit line to the material. If material is not included in the article's Creative Commons licence and your intended use is not permitted by statutory regulation or exceeds the permitted use, you will need to obtain permission directly from the copyright holder. To view a copy of this licence, visit http://creativecommons.org/licenses/by/4.0/.

\section{References}

1. Josephs KA, Dickson DW, Tosakulwong N, Weigand SD, Murray ME, Petrucelli L, et al. Rates of hippocampal atrophy and presence of post-mortem TDP-43 in patients with Alzheimer's disease: a longitudinal retrospective study. Lancet Neurol. 2017;16:917-24. https://doi.org/10.1016/s1474-4422(17)30284-3.

2. Braak H, Braak E. Neuropathological stageing of Alzheimerrelated changes. Acta Neuropathol. 1991;82:239-59. https://doi. org $/ 10.1007 /$ bf00308809.

3. McRae-McKee K, Evans S, Hadjichrysanthou C, Wong MM, de Wolf F, Anderson RM. Combining hippocampal volume metrics to better understand Alzheimer's disease progression in at-risk individuals. Sci Rep. 2019;9:7499. https://doi.org/10.1038/s41598-01942632-w.

4. Aggleton JP, Christiansen K. The subiculum: the heart of the extended hippocampal system. Prog Brain Res. 2015;219:65-82. https://doi.org/10.1016/bs.pbr.2015.03.003.

5. La C, Linortner P, Bernstein JD, Ua Cruadhlaoich MAI, Fenesy M, Deutsch GK, et al. Hippocampal CA1 subfield predicts episodic memory impairment in Parkinson's disease. Neuroimage Clin. 2019;23:101824. https://doi.org/10.1016/j.nicl.2019.101824.

6. Wintzer ME, Boehringer R, Polygalov D, McHugh TJ. The hippocampal CA2 ensemble is sensitive to contextual change. J Neurosci. 2014;34:3056-66. https://doi.org/10.1523/jneurosci.2563-13.2014.

7. Dalton MA, McCormick C, Maguire EA. Differences in functional connectivity along the anterior-posterior axis of human hippocampal subfields. Neuroimage. 2019;192:38-51. https://doi.org/10. 1016/j.neuroimage.2019.02.066.
8. Dalton MA, McCormick C, De Luca F, Clark IA, Maguire EA. Functional connectivity along the anterior-posterior axis of hippocampal subfields in the ageing human brain. Hippocampus. 2019;29:1049-62. https://doi.org/10.1002/hipo.23097.

9. Copara MS, Hassan AS, Kyle CT, Libby LA, Ranganath C, Ekstrom AD. Complementary roles of human hippocampal subregions during retrieval of spatiotemporal context. J Neurosci. 2014;34:6834-42. https://doi.org/10.1523/jneurosci.5341-13. 2014.

10. Greicius MD, Srivastava G, Reiss AL, Menon V. Default-mode network activity distinguishes Alzheimer's disease from healthy aging: evidence from functional MRI. Proc Natl Acad Sci U S A. 2004;101:4637-42. https://doi.org/10.1073/pnas.0308627101.

11. Li H, Jia X, Qi Z, Fan X, Ma T, Pang R, et al. Disrupted functional connectivity of cornu ammonis subregions in amnestic mild cognitive impairment: a longitudinal resting-state fMRI study. Front Hum Neurosci. 2018;12:413. https://doi.org/10.3389/fnhum.2018. 00413.

12. de Flores R, Mutlu J, Bejanin A, Gonneaud J, Landeau B, Tomadesso C, et al. Intrinsic connectivity of hippocampal subfields in normal elderly and mild cognitive impairment patients. Hum Brain Mapp. 2017;38:4922-32. https://doi.org/10.1002/hbm. 23704.

13. Stoub TR, de Toledo-Morrell L, Stebbins GT, Leurgans S, Bennett DA, Shah RC. Hippocampal disconnection contributes to memory dysfunction in individuals at risk for Alzheimer's disease. Proc Natl Acad Sci U S A. 2006;103:10041-5. https://doi.org/10.1073/pnas. 0603414103.

14. Platero C, Lopez ME, Carmen Tobar MD, Yus M, Maestu F. Discriminating Alzheimer's disease progression using a new hippocampal marker from T1-weighted MRI: the local surface roughness. Hum Brain Mapp. 2019;40:1666-76. https://doi.org/10. 1002/hbm.24478.

15. Choi EJ, Son YD, Noh Y, Lee H, Kim YB, Park KH. Glucose hypometabolism in hippocampal aubdivisions in Alzheimer's disease: a pilot study using high-resolution 18F-FDG PET and 7.0-T MRI. J Clin Neurol. 2018;14:158-64.

16. Barrett FS, Workman CI, Sair HI, Savonenko AV, Kraut MA, Sodums DJ, et al. Association between serotonin denervation and resting-state functional connectivity in mild cognitive impairment. Hum Brain Mapp. 2017;38:3391-401. https://doi.org/10.1002/ hbm.23595.

17. Adler DH, Wisse LEM, Ittyerah R, Pluta JB, Ding SL, Xie L, et al. Characterizing the human hippocampus in aging and Alzheimer's disease using a computational atlas derived from ex vivo MRI and histology. Proc Natl Acad Sci U S A. 2018;115:4252-7. https://doi. org/10.1073/pnas.1801093115.

18. Wang L, Zang Y, He Y, Liang M, Zhang X, Tian L, et al. Changes in hippocampal connectivity in the early stages of Alzheimer's disease: evidence from resting state fMRI. Neuroimage. 2006;31: 496-504. https://doi.org/10.1016/j.neuroimage.2005.12.033.

19. Cecchin D, Palombit A, Castellaro M, Silvestri E, Bui F, Barthel H, et al. Brain PET and functional MRI: why simultaneously using hybrid PET/MR systems? Q J Nucl Med Mol Imaging. 2017;61: 345-59. https://doi.org/10.23736/s1824-4785.17.03008-4.

20. McKhann GM, Knopman DS, Chertkow H, Hyman BT, Jack CR $\mathrm{Jr}$, Kawas CH, et al. The diagnosis of dementia due to Alzheimer's disease: recommendations from the National Institute on AgingAlzheimer's Association workgroups on diagnostic guidelines for Alzheimer's disease. Alzheimers Dement. 2011;7:263-9. https:// doi.org/10.1016/j.jalz.2011.03.005.

21. McKhann G, Drachman D, Folstein M, Katzman R, Price D, Stadlan EM. Clinical diagnosis of Alzheimer's disease: report of the NINCDS-ADRDA work group under the auspices of Department of Health and Human Services Task Force on 
Alzheimer's disease. Neurology. 1984;34:939-44. https://doi.org/ 10.1212/wnl.34.7.939.

22. Petersen RC. Mild cognitive impairment as a diagnostic entity. J Intern Med. 2004;256:183-94. https://doi.org/10.1111/j.13652796.2004.01388.x.

23. Vipin A, Loke YM, Liu S, Hilal S, Shim HY, Xu X, et al. Cerebrovascular disease influences functional and structural network connectivity in patients with amnestic mild cognitive impairment and Alzheimer's disease. Alzheimers Res Ther. 2018;10:82. https://doi.org/10.1186/s13195-018-0413-8.

24. Fazekas F, Chawluk JB, Alavi A, Hurtig HI, Zimmerman RA. MR signal abnormalities at $1.5 \mathrm{~T}$ in Alzheimer's dementia and normal aging. AJR Am J Roentgenol. 1987;149:351-6. https://doi.org/10. 2214/ajr.149.2.351.

25. Cui B, Zhang T, Ma Y, Chen Z, Ma J, Ma L, et al. Simultaneous PET-MRI imaging of cerebral blood flow and glucose metabolism in the symptomatic unilateral internal carotid artery/middle cerebral artery steno-occlusive disease. Eur J Nucl Med Mol Imaging. 2019. https://doi.org/10.1007/s00259-019-04551-w.

26. Shang K, Wang J, Fan X, Cui B, Ma J, Yang H, et al. Clinical value of hybrid TOF-PET/MR imaging-based multiparametric imaging in localizing seizure focus in patients with MRI-negative temporal lobe epilepsy. AJNR Am J Neuroradiol. 2018;39:1791-8. https:// doi.org/10.3174/ajnr.A5814.

27. Grant AM, Deller TW, Khalighi MM, Maramraju SH, Delso G, Levin CS. NEMA NU 2-2012 performance studies for the SiPMbased ToF-PET component of the GE SIGNA PET/MR system. Med Phys. 2016;43:2334. https://doi.org/10.1118/1.4945416.

28. Liu M, Paranjpe MD, Zhou X, Duy PQ, Goyal MS, Benzinger TLS, et al. Sex modulates the ApoE epsilon4 effect on brain tau deposition measured by (18)F-AV-1451 PET in individuals with mild cognitive impairment. Theranostics. 2019;9:4959-70. https://doi. org/10.7150/thno.35366.

29. Paranjpe MD, Chen X, Liu M, Paranjpe I, Leal JP, Wang R, et al. The effect of ApoE epsilon4 on longitudinal brain region-specific glucose metabolism in patients with mild cognitive impairment: a FDG-PET study. Neuroimage Clin. 2019;22:101795. https://oi. org/10.1016/j.nicl.2019.101795.

30. Tohka J, Reilhac A. Deconvolution-based partial volume correction in Raclopride-PET and Monte Carlo comparison to MR-based method. Neuroimage. 2008;39:1570-84. https://doi.org/10.1016/j. neuroimage.2007.10.038.

31. Chao-Gan Y, Yu-Feng Z. DPARSF:a MATLAB toolbox for "pipeline" data analysis of resting-state fMRI. Front Syst Neurosci. 2010;4:13. https://doi.org/10.3389/fnsys.2010.00013.

32. Eickhoff SB, Stephan KE, Mohlberg H, Grefkes C, Fink GR, Amunts K, et al. A new SPM toolbox for combining probabilistic cytoarchitectonic maps and functional imaging data. Neuroimage. 2005;25:1325-35. https://doi.org/10.1016/j.neuroimage.2004.12. 034.

33. Greg A, Holly B, Roderick MC, Hester AL, Fields JA, Weiner MF, et al. Reduced hippocampal functional connectivity in Alzheimer disease. Arch Neurol. 2007;64:1482-7. https://doi.org/10.1001/ archneur.64.10.1482.

34. Wang Z, Liang P, Jia X, Qi Z, Yu L, Yang Y, et al. Baseline and longitudinal patterns of hippocampal connectivity in mild cognitive impairment: evidence from resting state fMRI. J Neurol Sci. 2011;309:79-85. https://doi.org/10.1016/j.jns.2011.07.017.

35. de Flores R, La Joie R, Landeau B, Perrotin A, Mezenge F, de La Sayette V, et al. Effects of age and Alzheimer's disease on hippocampal subfields: comparison between manual and FreeSurfer volumetry. Hum Brain Mapp. 2015;36:463-74. https://doi.org/10. 1002/hbm.22640.

36. Tang X, Holland D, Dale AM, Miller MI. APOE affects the volume and shape of the amygdala and the hippocampus in mild cognitive impairment and Alzheimer's disease: age matters. J Alzheimers Dis. 2015;47:645-60. https://doi.org/10.3233/jad-150262.

37. Yassa MA, Stark SM, Bakker A, Albert MS, Gallagher M, Stark CE. High-resolution structural and functional MRI of hippocampal CA3 and dentate gyrus in patients with amnestic mild cognitive impairment. Neuroimage. 2010;51:1242-52. https://doi.org/10. 1016/j.neuroimage. 2010.03 .040 .

38. Pasquini L, Scherr M, Tahmasian M, Meng C, Myers NE, Ortner $\mathrm{M}$, et al. Link between hippocampus' raised local and eased global intrinsic connectivity in AD. Alzheimers Dement. 2015;11:475-84. https://doi.org/10.1016/j.jalz.2014.02.007.

39. Tahmasian M, Pasquini L, Scherr M, Meng C, Forster S, Mulej Bratec S, et al. The lower hippocampus global connectivity, the higher its local metabolism in Alzheimer disease. Neurology. 2015;84:1956-63. https://doi.org/10.1212/wnl.0000000000001575.

40. Gottler J, Preibisch C, Riederer I, Pasquini L, Alexopoulos P, Bohn KP, et al. Reduced blood oxygenation level dependent connectivity is related to hypoperfusion in Alzheimer's disease. J Cereb Blood Flow Metab. 2019;39:1314-25. https://doi.org/10.1177/0271678x18759182.

41. Bakker A, Krauss GL, Albert MS, Speck CL, Jones LR, Stark CE, et al. Reduction of hippocampal hyperactivity improves cognition in amnestic mild cognitive impairment. Neuron. 2012;74:467-74. https://doi.org/10.1016/j.neuron.2012.03.023.

42. Thorn CA, Popiolek M, Stark E, Edgerton JR. Effects of M1 and M4 activation on excitatory synaptic transmission in CA1. Hippocampus. 2017;27:794-810. https://doi.org/10.1002/hipo. 22732 .

43. von Gunten A, Kovari E, Bussiere T, Rivara CB, Gold G, Bouras C, et al. Cognitive impact of neuronal pathology in the entorhinal cortex and CA1 field in Alzheimer's disease. Neurobiol Aging. 2006;27:270-7. https://doi.org/10.1016/j.neurobiolaging.2005.02. 008 .

44. Adamowicz DH, Roy S, Salmon DP, Galasko DR, Hansen LA, Masliah E, et al. Hippocampal alpha-synuclein in dementia with Lewy bodies contributes to memory impairment and is consistent with spread of pathology. J Neurosci. 2017;37:1675-84. https://doi. org/10.1523/jneurosci.3047-16.2016.

45. Putcha D, Brickhouse M, O'Keefe K, Sullivan C, Rentz D, Marshall $\mathrm{G}$, et al. Hippocampal hyperactivation associated with cortical thinning in Alzheimer's disease signature regions in non-demented elderly adults. J Neurosci. 2011;31:17680-8. https://doi.org/10.1523/ jneurosci.4740-11.2011.

46. Thomas BA, Cuplov V, Bousse A, Mendes A, Thielemans K, Hutton BF, et al. PETPVC: a toolbox for performing partial volume correction techniques in positron emission tomography. Phys Med Biol. 2016;61:7975.

47. Jack CR Jr, Bennett DA, Blennow K, Carrillo MC, Dunn B, Haeberlein SB, et al. NIA-AA research framework: toward a biological definition of Alzheimer's disease. Alzheimers Dement. 2018;14:535-62. https://doi.org/10.1016/j.jalz.2018.02.018.

48. Xia M, Wang J, He Y. BrainNet viewer: a network visualization tool for human brain connectomics. PLoS One. 2013;8:e68910. https:// doi.org/10.1371/journal.pone.0068910.

Publisher's note Springer Nature remains neutral with regard to jurisdictional claims in published maps and institutional affiliations. 